\title{
Assessment of ultrasound and Doppler parameters in the third trimester of pregnancy as predictors of adverse perinatal outcome in unselected pregnancies
}

\author{
Alberto Borges Peixoto ${ }^{1}$, Taciana Mara Rodrigues da Cunha Caldas ${ }^{1}$, \\ Tácito Augusto Godoy Silva ${ }^{1}$, Mário Sérgio Silva Gomes Caetano ${ }^{1}$, Wellington P. Martins ${ }^{2}$, \\ Eduardo Felix Martins Santana ${ }^{3}$, Edward Araujo Júnior ${ }^{3}$ \\ ${ }^{1}$ Mario Palmério University Hospital — University of Uberaba, MG, Brazil \\ ${ }^{2}$ Department of Obstetrics and Gynecology, Ribeirão Preto Medical School, University of São Paulo, Ribeirão Preto, SP, Brazil \\ ${ }^{3}$ Department of Obstetrics, Paulista School of Medicine — Federal University of São Paulo, SP, Brazil
}

\begin{abstract}
Objectives: The aim of the study was to investigate ultrasound and Doppler parameters in the third trimester of pregnancy as possible predictors of adverse perinatal outcome in unselected pregnancies.

Material and methods: We performed a retrospective cross-sectional study including unselected pregnant women between 27 and $36+6$ weeks of gestation. The following ultrasound and Doppler parameters were assessed: estimated fetal weight (EFW) [g], EFW percentile, placental maturity grade (Grannum classification), single vertical deepest pocket (SVDP) of amniotic fluid [cm], amniotic fluid index (AFI) $[\mathrm{cm}]$, mean uterine artery $(\mathrm{UtA})$ pulsatility index (PI), umbilical artery (UA) $\mathrm{PI}$, middle cerebral artery (MCA) PI, MCA peak systolic velocity (PSV) $[\mathrm{cm} / \mathrm{s}]$, and cerebroplacental ratio (CPR). Adverse perinatal outcome was defined as Apgar score of $<7$ at $1 \mathrm{~min}$, birth weight of $<2500 \mathrm{~g}$ at delivery, and gestational age of $<37$ weeks at delivery. The unpaired $t$ test was used to compare the groups.

Results: AFI $(p=0.01)$, mean UtA PI $(p=0.04)$ and mean UA PI $(p=0.03)$ were significantly different with regard to the Apgar score at $1 \mathrm{~min}$. EFW, EFW percentile, SVDP of amniotic fluid, AFI, mean UtA PI, UA PI, and MCA PI were significantly different $(p<0.001)$ in terms of birth weight. Placental maturity grade $(p=0.02)$, SVDP of the amniotic fluid $(p<0.001)$, AFI $(p<0.001)$, mean UtA PI $(p<0.001)$, UA PI $(p=0.001)$, and MCA PI $(p<0.001)$ were significantly different as far as gestational age at delivery is concerned.

Conclusion: Ultrasound and Doppler parameters may predict adverse perinatal outcomes in unselected pregnancies in the third trimester of pregnancy.
\end{abstract}

Key words: ultrasound, Doppler, perinatal outcome, unselected, third trimester of pregnancy

Ginekologia Polska 2016; 87, 7: 510-515

\section{INTRODUCTION}

An ultrasound exam during pregnancy is essential to diagnose fetal malformations in the first and the second trimester [1], and to assess fetal growth disorders [2]. In the same way, Doppler ultrasound is vital for the assessment of intrauterine growth restriction (IUGR) [3], and as a predictor of preeclampsia in the first trimester of pregnancy [4].

The third-trimester ultrasound exam in high-risk pregnancies, i.e. borderline amniotic fluid volume and small for gestational age (SGA) fetuses, is necessary to predict adverse perinatal outcomes [5, 6]. Abnormal Doppler findings in the third trimester are typically associated with adverse perinatal outcomes $[7,8]$. However, the impact of a routine third-trimester ultrasound exam in unselected pregnancies seems poor, with high false-positive rates, leading to a steadily growing number of unnecessary elective cesarean sections [9]. Furthermore, ultrasound does not seem to be able to predict stillbirth [10]. However, some studies observed 
increasing adverse perinatal outcomes in unselected pregnancies presenting isolated low-normal early amniotic fluid volume [11], and increased maturity of the placental grade in smoking pregnant women [12].

\section{OBJECTIVES}

The aim of the study was to determine which ultrasound and Doppler parameters assessed in the third trimester ultrasound exams in unselected pregnancies could be predictors of adverse perinatal outcomes.

\section{MATERIAL AND METHODS}

We performed a retrospective cross-sectional study between April 2013 and June 2015 in unselected pregnant women undergoing a routine third-trimester ultrasound exam between 27 and $36+6$ weeks of gestation. Local Ethics Committee approved of the study. The consent form was unnecessary due to the retrospective nature of the study. The pregnant women were recruited from public and private health services of the urban region of Uberaba (MG, Brazil).

The inclusion criteria were as follows: 1) singleton pregnancy, 2) gestational age determined by the last menstrual period (LMP) and confirmed by first-trimester ultrasound using crown-rump length (CRL) until 13 weeks of gestation, 3) absence of fetal structural malformations or chromosomal abnormalities on ultrasound and confirmed in the postnatal period, 4) delivery at the Mário Palmério University Hospital.

All ultrasound exams were performed at the Mário Palmério University Hospital by two examiners (ABP and TMRCC), with Fetal Medicine Foundation (FMF) accreditation, using a Voluson E6 (General Electric, Zipf, Austria) equipped with a convex (RAB4-6L) and an endovaginal (RIC5-9W) probe. The following ultrasound and Doppler parameters were assessed: estimated fetal weight (EFW), EFW percentile, placental maturity grade, single deepest vertical pocket (SVDP) of amniotic fluid, amniotic fluid index (AFI), mean UtA pulsatility index (PI), mean UA PI, mean middle cerebral artery (MCA) PI, MCA peak systolic velocity (PSV), and cerebroplacental ratio (CPR). EFW [g] was calculated automatically by the apparatus using Hadlock's formula [13]:

Log 10 birth weight $=1.4787+0.001837 \times$ (biparietal diameter $)^{2}+0.0458 \times($ abdominal circumference $)+0.158 \times(\mathrm{fe}-$ mur length) $-0.003343 \times$ (abdominal circumference $\times$ femur length).

EFW percentile was calculated according to the gestational age using Hadlock's chart [14]. The Grannum classification was used to evaluate placental maturity grade [15]. According to this classification, the placental maturity grade ranges from 0 to 3, depending on specific ultrasonic findings at the basal and chorionic plates as well as within the substance of the organ itself, with 0 signifying the least mature placenta and absence of basal plate calcifications, and 3 indicating the most mature placenta and calcifications within the placenta forming cotyledons. SVDP of amniotic fluid was measured by positioning the probe vertically to the uterine contour of the abdomen and parallel to the maternal sagittal plane, free of small parts or umbilical cord [16]. AFI was calculated in agreement with Phelan et al. [17], in other words, the maternal abdomen was divided into four quadrants at the level of the umbilicus and the AFI measurement was obtained by adding the four deepest vertical pockets of amniotic fluid. UtA Doppler was performed transabdominally. The probe was placed in the lower quadrant of the abdomen, angled medially, and color Doppler imaging was used to identify UtA at the apparent crossover with the external iliac artery. Measurements were taken approximately $1.0 \mathrm{~cm}$ distally to the crossover point. In all cases, once it had been ensured that the angle was $<30^{\circ}$, the pulsed Doppler gate was placed over the whole width of the vessel. Angle correction was then applied and the signal was updated until three similar consecutive waveforms were obtained. Left and right artery PI values were measured, and mean PI was calculated [18]. UA PI was obtained using color Doppler to identify the vessel in the placental end of the umbilical cord. The pulsed-wave Doppler gate was positioned in one of the umbilical arteries with the insonation angle of $<30^{\circ}$ and the sample volume encompassed the entire width of the vessel. If three similar consecutive waveforms were obtained, mean PI of these waveforms was used for the analysis [19]. We assessed MCA PI in the axial plane of the brain, including the thalamic and the sphenoid bone wings. Color Doppler was used to identify the circle of Willis and the proximal MCA. Pulsed-wave Doppler gate was positioned in the proximal third of MCA, close to its origin in the internal carotid artery, with sample volume around the entire width of the vessel and insonation angle of $<30^{\circ}$. When three similar consecutive waveforms were obtained, mean PI of these waveforms was used for the analysis [20]. At this level, PSV [cm/s] as the highest point of the waveform, was calculated [20]. CPR was obtained using the following ratio: mean PI MCA / mean PI UA [21].

We defined an adverse perinatal outcome as Apgar score of $<7$ at $1 \mathrm{~min}$, birth weight of $<2500 \mathrm{~g}$ at delivery, and gestational age of $<37$ weeks at delivery. In cases of repeated ultrasound exams of the same pregnant woman, we took into account the first exam, if it was performed between 27 and $36+6$ weeks of gestation.

Data were transferred to an Excel spread sheet (Microsoft Corp., Redmond, WA, EUA) and analyzed by one of the authors (WPM) using PASW software (version 18.0, SPSS Inc., Chicago, IL, USA). Maternal characteristics such as age, height, weight, body mass index (BMI), gravidity, parity and gestational age at ultrasound exam, gestational age at delivery and birth weight, and Apgar scores at 1 and 5 min, were expressed as 
mean \pm standard deviation (SD). EFW, EFW percentile, placental maturity grade, SVDP of amniotic fluid, AFI, mean UtA PI, mean UA PI, mean MCA PI, MCA PSV, and CPR were expressed as mean $\pm S D$ and compared the perinatal outcome groups (Apgar score at $1 \mathrm{~min}$ of $\geq 7 \mathrm{vs.}<7$; birth weight of $\geq 2500 \mathrm{~g}$ vs. $<2500$ g; and gestational age of $\geq 37$ weeks vs. $<37$ weeks at delivery) using the unpaired $t$ test. The $p$-value of $<0.05$ was considered as statistically significant.

\section{RESULTS}

In our database, a total of 2157 ultrasound exams were selected during the study period. However, 1457 exams were excluded because of the following reasons: $622<27$ weeks of gestation, $417 \geq 38$ weeks of gestation, 64 lost to follow-up and 354 underwent repeated ultrasound exams. Therefore, we analyzed data from $700 \mathrm{ul}$ trasound scans: 103 pregnant women (14.7\%) were considered high-risk because of the following maternal chronic diseases: genetic thrombophilia (2), hypothyroidism (32), preeclampsia (12), chronic hypertension (27), epilepsy (1), type 1 diabetes mellitus (1), type 2 diabetes mellitus (1), gestational diabetes (21), chronic hypertension and gestational diabetes (1), Chagas disease (1), asthma (2), and anemia (2). The remaining 597 (85.3\%) pregnant women were considered low-risk patients.

When comparing the results depending on the Apgar score at $1 \mathrm{~min}$, we excluded data from 82 pregnancies without data for these outcomes. Therefore, we compared 618 pregnancies depending on the Apgar score at $1 \mathrm{~min}: \geq 7(n=565)$ vs. $<7(n=53)$. Table 1 shows a comparison of the 1-min. Apgar score of $\geq 7$ vs. $<7$ and maternal characteristics. We observed significant differences in gestational age at delivery and birth weight parameters. Table 2 shows a comparison of the 1-min Apgar score of $\geq 7$ vs. $<7$ with regard to the ultrasound and Doppler parameters. We observed significant differences in AFI, mean UtA PI. and UA PI.

Table 1. Comparison between Apgar score of $\geq 7 \mathrm{vs} .<7$ at $1 \mathrm{~min}$ with regard to maternal characteristics

\begin{tabular}{|c|c|c|c|c|c|c|c|}
\hline \multirow[b]{2}{*}{ Parameter } & \multicolumn{3}{|c|}{ Apgar $1^{\text {st }} \geq 7$} & \multicolumn{3}{|c|}{ Apgar $1^{\text {st }}<7$} & \multirow{2}{*}{$\mathbf{p}$} \\
\hline & $\mathrm{n}$ & Mean & SD & $\mathbf{n}$ & Mean & SD & \\
\hline Age [years] & 565 & 26.3 & 6.5 & 53 & 24.9 & 6.4 & 0.13 \\
\hline Weight [kg] & 478 & 74.0 & 18.0 & 42 & 70.9 & 14.6 & 0.27 \\
\hline Height [cm] & 400 & 161.4 & 7.1 & 26 & 161.6 & 6.4 & 0.91 \\
\hline BMI $\left[\mathrm{kg} / \mathrm{m}^{2}\right]$ & 400 & 28.4 & 7.1 & 26 & 28.7 & 6.0 & 0.80 \\
\hline Gravidity & 492 & 2.2 & 1.5 & 43 & 2.2 & 2.1 & 0.83 \\
\hline Parity & 492 & 1.0 & 1.2 & 43 & 1.0 & 1.9 & 0.66 \\
\hline GA at scan [weeks] & 565 & 33.6 & 2.5 & 53 & 33.5 & 2.6 & 0.63 \\
\hline GA at delivery [weeks] & 565 & 38.3 & 2.3 & 53 & 36.5 & 3.3 & $<0.001^{*}$ \\
\hline Birth weight [g] & 565 & 3015.8 & 543.9 & 51 & 2609.1 & 863.4 & $<0.001^{*}$ \\
\hline
\end{tabular}

*Unpaired t test; SD — standard deviation; BMI — body mass index; GA — gestational age

Table 2. Comparison between Apgar score at $1 \mathrm{~min} \geq 7$ vs. $<7$ perinatal outcomes with regard to ultrasound and Doppler parameters

\begin{tabular}{|c|c|c|c|c|c|c|c|}
\hline \multirow[b]{2}{*}{ Parameter } & \multicolumn{3}{|c|}{ Apgar $1^{\text {st }} \geq 7(n=565)$} & \multicolumn{3}{|c|}{ Apgar $1^{\text {st }}<7(n=53)$} & \multirow{2}{*}{$\mathbf{p}$} \\
\hline & $\mathbf{n}$ & Mean & SD & $n$ & Mean & SD & \\
\hline $\mathrm{EFW}[\mathrm{g}]$ & 547 & $2,200.5$ & 556.2 & 52 & $2,161.3$ & 641.1 & 0.63 \\
\hline EFW (percentile) & 547 & 40.3 & 24.6 & 52 & 41.5 & 28.3 & 0.75 \\
\hline Placenta (Grannum) & 487 & 1.7 & 0.6 & 47 & 1.7 & 0.5 & 0.53 \\
\hline $\operatorname{SVDP}[\mathrm{cm}]$ & 479 & 4.7 & 1.5 & 43 & 5.1 & 2.2 & 0.09 \\
\hline $\mathrm{AFI}[\mathrm{cm}]$ & 256 & 11.1 & 4.0 & 30 & 13.5 & 7.4 & $0.01^{*}$ \\
\hline Uterine artery PI & 242 & 0.77 & 0.26 & 15 & 0.98 & 0.45 & $0.004^{*}$ \\
\hline Umbilical artery PI & 419 & 0.93 & 0.25 & 38 & 1.04 & 0.66 & $0.03^{*}$ \\
\hline MCAPI & 369 & 1.8 & 0.4 & 31 & 1.7 & 0.4 & 0.05 \\
\hline MCA PSV $[\mathrm{cm} / \mathrm{s}]$ & 325 & 52.6 & 10.3 & 29 & 54.3 & 8.7 & 0.40 \\
\hline CPR & 419 & 1.8 & 0.8 & 38 & 1.5 & 0.9 & 0.05 \\
\hline
\end{tabular}

*Unpaired t test; SD — standard deviation; EFW — estimated fetal weight; SVDP — single vertical deepest pocket; AFI — amniotic fluid index; PI — pulsatility index; MCA — middle cerebral artery; PSV — peak systolic velocity; CPR — cerebroplacental ratio 
Table 3. Comparison between birth weight of $\geq 2500 \mathrm{~g}$ vs. $<2500 \mathrm{~g}$ perinatal outcomes with regard to ultrasound and Doppler parameters

\begin{tabular}{|l|c|c|c|c|c|c|c|c|}
\hline & \multicolumn{3}{|c|}{ Birth weight $\geq \mathbf{2 5 0 0} \mathbf{g}(\mathbf{n}=\mathbf{5 6 5})$} & \multicolumn{3}{c|}{ Birth weight $<\mathbf{2 5 0 0} \mathbf{g}(\mathbf{n}=\mathbf{1 3 0})$} \\
\hline Parameter & $\mathbf{n}$ & Mean & $\mathbf{S D}$ & $\mathbf{n}$ & $\mathbf{M e a n}$ & $\mathbf{S D}$ & $\mathbf{p}$ \\
\hline EFW [g] & 546 & $2,242.57$ & 564.46 & 126 & $1,903.93$ & 509.58 & $<0.001^{*}$ \\
\hline EFW (percentile) & 546 & 43.39 & 23.81 & 125 & 26.27 & 25.27 & $<0.001^{*}$ \\
\hline Placenta (Grannum) & 478 & 1.67 & 0.61 & 126 & 1.72 & 0.61 & 0.43 \\
\hline SVDP [cm] & 474 & 4.84 & 1.47 & 117 & 4.07 & 1.69 & $<0.001^{*}$ \\
\hline AFI [cm] & 261 & 11.79 & 4.11 & 65 & 9.28 & 5.11 & $<0.001^{*}$ \\
\hline Uterine artery PI & 232 & 0.74 & 0.20 & 60 & 0.97 & 0.44 & $<0.001^{*}$ \\
\hline Umbilical artery PI & 394 & 0.91 & 0.15 & 119 & 1.05 & 0.54 & $<0.001^{*}$ \\
\hline MCA PI & 337 & 1.84 & 0.36 & 115 & 1.69 & 0.40 & $<0.001^{*}$ \\
\hline MCA PSV [cm/s] & 300 & 53.16 & 10.41 & 101 & 51.23 & 9.89 & 0.10 \\
\hline CPR & 394 & 1.75 & 0.85 & 119 & 1.74 & 0.69 & 0.86 \\
\hline
\end{tabular}

*Unpaired t test; SD — standard deviation; EFW — estimated fetal weight; SVDP — single vertical deepest pocket; AFI — amniotic fluid index; PI — pulsatility index; MCA — middle cerebral artery; PSV — peak systolic velocity; CPR — cerebroplacental ratio

\begin{tabular}{|c|c|c|c|c|c|c|c|}
\hline \multirow[b]{2}{*}{ Parameter } & \multicolumn{3}{|c|}{ Delivery $\geq 37$ weeks $(n=512$ ) } & \multicolumn{3}{|c|}{ Delivery < 37 weeks $(n=188$ ) } & \multirow[b]{2}{*}{$\mathbf{p}$} \\
\hline & n & Mean & SD & $\mathbf{n}$ & Mean & SD & \\
\hline EFW [g] & 494 & $2,179.60$ & 549.76 & 183 & $2,169.73$ & 625.41 & 0.84 \\
\hline EFW (percentile) & 494 & 40.02 & 22.92 & 182 & 40.83 & 30.00 & 0.71 \\
\hline Placenta (Grannum) & 430 & 1.64 & 0.59 & 179 & 1.78 & 0.65 & $0.02^{*}$ \\
\hline $\operatorname{SVDP}[\mathrm{cm}]$ & 428 & 4.87 & 1.40 & 168 & 4.22 & 1.77 & $<0.001^{*}$ \\
\hline AFI [cm] & 222 & 12.05 & 3.99 & 106 & 9.65 & 4.85 & $<0.001^{*}$ \\
\hline Uterine artery PI & 233 & 0.75 & 0.20 & 63 & 0.93 & 0.44 & $<0.001^{*}$ \\
\hline Umbilical artery PI & 350 & 0.91 & 0.15 & 167 & 1.00 & 0.47 & $0.001^{*}$ \\
\hline MCA PI & 298 & 1.86 & 0.36 & 158 & 1.68 & 0.38 & $<0.001^{*}$ \\
\hline MCA PSV $[\mathrm{cm} / \mathrm{s}]$ & 278 & 53.22 & 10.63 & 127 & 51.33 & 9.39 & 0.09 \\
\hline CPR & 350 & 1.76 & 0.87 & 167 & 1.75 & 0.71 & 0.883 \\
\hline
\end{tabular}

*Unpaired t test; SD — standard deviation; EFW — estimated fetal weight; SVDP — single vertical deepest pocket; AFI — amniotic fluid index; PI — pulsatility index; MCA — middle cerebral artery; PSV — peak systolic velocity; CPR — cerebroplacental ratio

As far as birth weight is concerned, we excluded 5 more cases because these values were not available. Therefore, we compared birth weight of $\geq 2500 \mathrm{~g}(\mathrm{n}=565) \mathrm{vs}$. $<2500 \mathrm{~g}$ ( $\mathrm{n}=130$ ) from 695 pregnancies. Table 3 shows the comparison between the birth weight of $\geq 2500 \mathrm{~g}$ vs. $<2.500 \mathrm{~g}$. We observed significant differences in EFW, EFW percentile, SVDP of amniotic fluid, AFI, UtA PI, UA PI, and MCA PI parameters.

We excluded 10 more cases from the analysis of gestational age at delivery because the values of this variable were not available. Therefore, we compared a total of 695 pregnancies depending on gestational age of $\geq 37$ weeks ( $n=512$ ) vs. $<37$ weeks $(n=188)$ at delivery. Table 4 shows a comparison between gestational age of $\geq 37$ weeks vs. $<37$ weeks at delivery. We observed significant differences in placental maturity grade, SVDP of amniotic fluid, AFI, mean UtA PI, mean UA PI, and mean MCA PI parameters.

\section{DISCUSSION}

Our study assessed the capacity of ultrasound and Doppler parameters as predictors of adverse perinatal outcomes in unselected pregnancies in the third trimester. In our prospective study including both, high- and low-risk pregnancies with altered UtA Doppler between 27 and 41 weeks of gestation, adverse perinatal outcomes (SGA, cesarean section and low Apgar scores) were associated with both, lowand high-risk pregnancies with altered UtA Doppler [8]. Cesarean section was not considered to be an adverse perinatal outcome in our study because this surgery rate is very high in Brazil, mainly due to the fact that private hospitals (86.2\%) perform the procedure without obstetric indications [22]. In our study, uterine artery Doppler evaluation was associated with Apgar score of $<7$ at $1 \mathrm{~min}$, birth weight of $<2500 \mathrm{~g}$, and gestational age of $<37$ weeks at delivery, proving the 
importance of the assessment of this Doppler index in the third trimester in low-risk and unselected populations. Kwon et al. [7], observed a 5-fold higher risk of an adverse perinatal outcome when abnormal Doppler was associated with borderline AFI $(5-8 \mathrm{~cm})$ in 3740 unselected pregnancies. In South Korea, Doppler velocimetry is a routine test in the third trimester of pregnancy, regardless of the risk [7]. In Brazil, Doppler velocimetry is not routine in prenatal care, which explains why only $42 \%$ of the pregnant women from our study underwent this exam.

The third trimester ultrasound exam is performed to assess fetal growth in low-risk pregnancies, as well as the follow-up of high-risk pregnancies by assessing AFI, UA Doppler and the biophysical profile. In our study, we used an unselected population, including both, low- and high-risk pregnancies, in order to try and predict adverse perinatal outcomes using ultrasound and Doppler parameters which are typically assessed in the third trimester. Oligohydramnios has been proven to be a reliable marker of adverse perinatal outcome [23]. However, monitoring of the amniotic fluid volume in low-risk pregnancies remains controversial. Hashimoto et al. [11], in their retrospective cohort study, assessed two groups of low-risk pregnant women (low-normal AFI $8-11 \mathrm{~cm}$ ) and (mid-normal AFI 12-19.9 cm) between 28 and 31.9 weeks of gestation and observed that the low-normal AFI group had higher preterm delivery and SGA rates. In another study, involving 3740 unselected pregnant women within two weeks before the delivery, the borderline AFI group $(5-8 \mathrm{~cm}$ ) presented a 3 -fold increase in the incidence of adverse perinatal outcomes as compared to the normal AFI group (> $8 \mathrm{~cm}$ ). It remains controversial which would be the best parameter to assess the amniotic fluid volume during pregnancy. In a systematic review of randomized controlled trials including both, low- and high-risk pregnancies, SVDP was a better choice than AFI due to the fact that the latter increased oligohydramnios and induction of labor rates without an improvement in the perinatal outcome [24]. In our study, AFI was associated with the 1-min Apgar score of $<7$, birth weight of $<2500 \mathrm{~g}$, and gestational age of $<37$ weeks at delivery, while SVDP was associated with the last two perinatal outcomes. However, small simple size could have interfered with the results. We believe that the follow-up of amniotic fluid volume in the third trimester using AFI or SVDP is important in order to prevent adverse perinatal outcomes in low-risk and unselected pregnancies.

Regarding prediction of SGA fetuses (EFW $<10^{\text {th }} \mathrm{Had}$ lock's formula [25]) during third-trimester ultrasound, in a French study which involved 2002 low-risk pregnancies, the ultrasound exam had a sensitivity of $29 \%$ and positive predictive value of $30.8 \%$, increasing the planned preterm delivery and elective cesarean section in false-positive non-SGA neonates [9]. In our study, EFW and EFW percentile were associated only with birth weight of $<2500 \mathrm{~g}$. On the other hand, Souka et al. [26], performed a cross-sectional study including 2310 low-risk pregnancies between 30 and 34 weeks of gestation to evaluate the performance of ultrasound in the prediction of SGA fetuses $\left(E F W<10^{\text {th }}\right.$ Hadlock's formula) [25]. These authors demonstrated that EFW is a good predictor of SGA (area under the receiver-operating characteristics $=0.87$ ). Hadlock's formulas $[13,15]$ have been used worldwide. However, the ethic factor may affect the fetal biometric parameter measurements, e.g. fetuses in Morocco and Turkey had smaller abdominal circumference, head circumference, and femur length measurements as compared to the Belgian population [27].

The Grannum classification was used to evaluate placental maturity grade [15]. In a prospective study of 1011 low-risk pregnancies, placental maturity (Grannum) — assessed between 22 and 36 weeks of gestation - was associated with threatened first- or second-trimester pregnancy loss, preeclampsia, and birth weight of $<9^{\text {th }}$ centile [28]. In another study of 1802 low-risk pregnancies, identification of Grannum grade III placenta at 36 weeks of gestation was associated with preeclampsia and birth weight of $<10^{\text {th }}$ percentile [29]. In our study, placental maturity grade (Grannum classification) was associated only with gestational age at delivery as perinatal outcome. We believe that small sample size and the fact that we used an unselected population could have influenced the findings of our study.

We did not analyze umbilical cord blood PH after delivery due to the fact that our unselected-population consisted of 597 (85.3\%) low-risk pregnancies. In a previous study, which included 47 low-risk pregnant women, no significant correlations between Apgar score at 1 and $5 \mathrm{~min}$, and umbilical cord $\mathrm{pH}$ in the low-risk group were found [30].

Our study was not without limitations, namely a relatively small simple size and only one ultrasound scan per each pregnant woman. However, as only two experienced examiners with FMF accreditation performed the ultrasound exams, the variability on the ultrasound and Doppler parameter measurements was reduced. Furthermore, we used an unselected population (including both, low- and high-risk pregnancies), which could have interfered with the results, although the main objective of the study was to use the most heterogeneous population possible to find the real ultrasound and Doppler parameters to predict adverse perinatal outcomes.

\section{CONCLUSIONS}

Ultrasound and Doppler parameters, mainly AFI and umbilical and uterine arteries $\mathrm{PI}$, may predict adverse perinatal outcomes in unselected pregnancies in the third trimester of pregnancy. 


\section{REFERENCES}

1. Mandruzzato G, Meir YJ, Natale R, [et al.]. Antepartal assessment of IUGR fetuses. J Perinat Med. 2001, 29, 222-223.

2. Levi S. Ultrasound in prenatal diagnosis: polemics around routine ultrasound screening for second trimester fetal malformations. Prenat Diagn. 2002, 22, 285-295.

3. Botsis D, Vrachnis N, Christodoulakos G. Doppler assessment of the intrauterine growth-restricted fetus. Ann N Y Acad Sci. 2006, 1092, 297-303.

4. Crovetto F, Figueras F, Triunfo $S$, [et al.]. First trimester screening for early and late preeclampsia based on maternal characteristics, biophysical parameters, and angiogenic factors. Prenat Diagn. 2015, 35, 183-191.

5. Gumus II, Koktener A, Turhan NO. Perinatal outcomes of pregnancies with borderline amniotic fluid index. Arch Gynecol Obstet. 2007, 276, 17-19.

6. Magann EF, Haas DM, Hill JB, [et al.]. Oligohydramnios, small for gestational age and pregnancy outcomes: an analysis using precise measures. Gynecol Obstet Invest. 2011, 72, 239-244.

7. Kwon JY, Kwon HS, Kim YH, Park YW. Abnormal Doppler velocimetry is related to adverse perinatal outcome for borderline amniotic fluid index during third trimester. J Obstet Gynaecol Res. 2006, 32, 545-549.

8. Shwarzman P, Waintraub AY, Frieger M, [et al.]. Third-trimester abnormal uterine artery Doppler findings are associated with adverse pregnancy outcomes. J Ulrasound Med. 2013, 32, 2107-2113.

9. Callec R, Lamy C, Perdriolle-Galet E, [et al.]. Impact on obstetric outcome of third-trimester screening for small-for-gestational-age fetuses. Ultrasound Obstet Gynecol 2014 [ahead of print].

10. Mone F, Meti S, Ong S. Does performing fetal ultrasound assessment once versus twice in the third trimester in low risk women alter the stillbirth rate? Ir Med J. 2014, 107, 181-183.

11. Hashimoto $K$, Kasdaglis $T$, Jain $\mathrm{S}$, [et al.]. Isolated low-normal amniotic fluid volume in the early third trimester: association with adverse perinatal outcomes. J Perinat Med. 2013, 41, 349-353.

12. Walker MG, Hindmarsh PC, Geary M, Ket alingdom JC. Sonographic maturation of the placenta at 30 to 34 weeks is not associated with second trimester markers of placental insufficiency in low-risk pregnancies. J Obstet Gynaecol Can. 2010, 32, 1134-1139.

13. Hadlock FP, Harrist RB, Carpenter RJ, [et al.]. Sonographic estimation of fetal weight. The value of femur length in addition to head and abdomen measurements. Radiology. 1984, 150, 535-540.

14. Hadlock FP, Harrist RB, Martinez-Poyer J. In utero analysis of fetal growth: a sonographic weight standard. Radiology. 1991, 181, 129-133.

15. Grannum PA, Berkowitz RL, Hobbins JC. The ultrasonic changes in the maturing placenta and their relation to fetal pulmonic maturity. Am J Obstet Gynecol. 1979, 133, 915-922.

16. Gramellini D, Chiaie D, Piantelli G, [et al.]. Sonographic assessment of amniotic fluid volume between 11 and 24 weeks of gestation: construc- tion of reference intervals related to gestational age. Ultrasound Obstet Gynecol. 2001, 17, 410-415.

17. Phelan JP, Ahn MO, Smith CV, [et al.]. Amniotic fluid index measurements during pregnancy. J Reprod Med. 1987, 32, 601-604.

18. Gómez $\mathrm{O}$, Figueras F, Fernándes $\mathrm{S}$, [et al.]. Reference ranges for uterine artery mean pulsatility index at 11-41 weeks of gestation. Ultrasound Obstet Gynecol. 2008, 32, 128-132.

19. Acharya G, Wilsgaard T, Berntsen GK, [et al.]. Reference ranges for serial measurements of blood velocity and pulsatility index at the intra-abdominal portion, and fetal and placental ends of the umbilical artery. Ultrasound Obstet Gynecol. 2005, 26, 162-169.

20. Mari G, Deter RL, Carpenter RL, [et al.]. Noninvasive diagnosis by Doppler ultrasonography of fetal anemia due to maternal red-cell alloimmunization. Collaborative Group for Doppler Assessment of the Blood Velocity in Anemic Fetuses. N Engl J Med. 2000, 342, 9-14.

21. Gramellini D, Folli MC, Raboni S, [et al.]. Cerebral-umbilical Doppler ratio as a predictor of adverse perinatal outcome. Obstet Gynecol. 1992, 79, 416-420.

22. Vieira GO, Fernandes LG, de Oliveira NF, [et al.]. Factors associated with cesarean delivery in public and private hospitals in a city of northeastern Brazil: a cross-sectional study. BMC Pregnancy Childbirth. 2015, 15, 132.

23. Vink J, Hickey K, Ghidini A, [et al.]. Earlier gestational age at ultrasound evaluation predicts adverse neonatal outcomes in the preterm appropriate-for-gestational age fetus with idiopathic oligohydramnios. Am J Perinatol. 2009, 26, 21-25.

24. Nabhan AF, Abdelmoula YA. Amniotic fluid index versus single deepest vertical pocket as a screening test for preventing adverse pregnancy outcome. Cochrane Database Syst Rev. 2008, 3, CD006596.

25. Hadlock FP, Harrist RB, Sharman RS, [et al.]. Estimation of fetal weight with the use of head, body, and femur measurements - a prospective study. Am J Obstet Gynecol. 1985, 151, 333-337.

26. Souka AP, Papastefanou I, Pilalis A, [et al.]. Performance of third-trimester ultrasound for prediction of small-for-gestational-age neonates and evaluation of contingency screening policies. Ultrasound Obstet Gynecol. 2012, 39, 535-542.

27. Jacquemyn $\mathrm{Y}$, Sys SU, Verdonk P. Fetal biometry in different ethnic groups. Early Hum Dev. 2000, 57, 1-13.

28. Cooley SM, Donnelly JC, Walsh T, [et al.]. The impact of ultrasonographic placental architecture on antenatal course, labor and delivery in a low-risk primigravid population. J Matern Fetal Neonatal Med. 2011, 24, 493-497.

29. McKenna D, Tharmaratnam S, Mahsud S, [et al.]. Ultrasonic evidence of placental calcification at 36 weeks' gestation: maternal and fetal outcomes. Acta Obstet Gynecol Scand. 2005, 84, 7-10.

30. Ahmadpour-Kacho M, Asnafi N, Javadian M, [et al.]. Correlation between Umbilical Cord pH and Apgar Score in High-Risk Pregnancy. Iran J Pediatr. 2010, 20, 401-406. 